Gluteus maximus hypertrophy : A diagnostic clue in four and a half LIM domain 1-mutated reducing body myopathy

Jokela, Manu

$2017-10$

Jokela , M , Huovinen , S , Palmio , J , Saukkonen , A-M , Penttila , S \& Udd , B 2017 , '

Gluteus maximus hypertrophy : A diagnostic clue in four and a half LIM domain 1-mutated

reducing body myopathy ' , Neuromuscular Disorders , vol. 27 , no. 10 , pp. 962-963 . https://doi.org/10.1016/j.nmd.2

http://hdl.handle.net/10138/298126

https://doi.org/10.1016/j.nmd.2017.06.014

publishedVersion

Downloaded from Helda, University of Helsinki institutional repository.

This is an electronic reprint of the original article.

This reprint may differ from the original in pagination and typographic detail.

Please cite the original version. 


\title{
Gluteus maximus hypertrophy: A diagnostic clue in four and a half LIM domain 1-mutated reducing body myopathy
}

\author{
Manu Jokela a,b,*, Sanna Huovinen ${ }^{c}$, Johanna Palmio ${ }^{b}$, Anna-Maija Saukkonen ${ }^{\mathrm{d}}$, Sini Penttilä ${ }^{\mathrm{b}}$, \\ Bjarne Udd ${ }^{\text {bee,f }}$ \\ ${ }^{a}$ Division of Clinical Neurosciences, Turku University Hospital and University of Turku, Turku, Finland \\ ${ }^{\mathrm{b}}$ Neuromuscular Research Center, Tampere University Hospital and University of Tampere, Tampere, Finland \\ ${ }^{\mathrm{c}}$ Department of Pathology, Fimlab Laboratories, Tampere University Hospital and University of Tampere, Tampere, Finland \\ ${ }^{\mathrm{d}}$ Department of Neurology, Central Hospital of Northern Karelia, Joensuu, Finland \\ ${ }^{\mathrm{e}}$ Department of Neurology, Vasa Central Hospital, Vasa, Finland \\ ${ }_{\mathrm{f}}^{\mathrm{f}}$ Folkhälsan Genetic Institute, Department of Medical Genetics, Helsinki University, Helsinki, Finland \\ Received 20 February 2017; received in revised form 16 May 2017; accepted 19 June 2017
}

Keywords: Reducing body myopathy; Magnetic resonance imaging; FHL1

A 28-year old male was examined due to progressive proximodistal muscle weakness of three years' duration. Severe generalized skeletal muscle weakness and atrophy spared only the facial and distal hand muscles. The patient had marked neck rigidity and scapular winging. Echocardiography was normal. Over the following three years, weakness progressed rapidly and the patient became wheelchair-bound. Muscle MRI at age 29 showed severe fatty degeneration of the thigh and lower leg muscles predominantly involving the posterior compartments, whereas gluteus maximus muscles were markedly hypertrophic (Fig. 1A). A muscle biopsy (vastus lateralis) showed severe dystrophic changes and large cytoplasmic bodies reacting strongly with menadione-NBT (Fig. 1B). On electron microscopy these reducing bodies were composed of granulofilamentous material (Fig. 1C). FHL1 gene sequencing revealed a previously unreported hemizygous mutation c.301T $>\mathrm{C}$ causing an amino acid change C100R in the second LIM domain of FHL1 protein.

The spectrum of X-dominant FHL1-opathies can be broadly divided into two categories: (1) Reducing body myopathies (RBM) with FHL1 mutations affecting all three FHL1 isoforms, usually missense mutations, and (2) Emery-Dreifuss-like muscular dystrophies without reducing bodies on muscle biopsy, usually caused by truncating loss-of-protein mutations affecting one or two FHL1 isoforms [1]. Predominantly scapuloperoneal weakness, axial rigidity and selective muscle hypertrophy or pseudoathleticism are commonly observed in both types [1-3].

Recently, a distinctive MRI pattern including preserved or hypertrophic gluteus maximus muscles was described in FHL1linked RBM based on three families with FHL1 mutations C153R, C153Y and C150S [2,3]. We observed similar muscle involvement with striking gluteus maximus hypertrophy in our patient with a novel FHL1 mutation (C100R) and reducing body muscle pathology. This peculiar MRI finding may be a useful clinical clue to suggest an FHL1-related RBM disease. Although not routinely reported, the inclusion of pelvic muscles may add to the usefulness of MRI pattern recognition.

\section{References}

[1] Schessl J, Feldkirchner S, Kubny C, Schoser B. Reducing body myopathy and other FHL1-related muscular disorders. Semin Pediatr Neurol 2011;18 (4):257-63.

[2] Astrea G, Schessl J, Clement E, Tosetti M, Mercuri E, Rutherford M, et al. Muscle MRI in FHL1-linked reducing body myopathy. Neuromuscul Disord 2009;19(10):689-91.

[3] Schreckenbach T, Henn W, Kress W, Roos A, Maschke M, Feiden W, et al. Novel FHL1 mutation in a family with reducing body myopathy. Muscle Nerve 2013;47(1):127-34.

\footnotetext{
* Corresponding author. Division of Clinical Neurosciences, Turku University Hospital and University of Turku, Kiinamyllynkatu 4-8, 20520 Turku, Finland. E-mail address: mejoke@utu.fi (M. Jokela).
} 


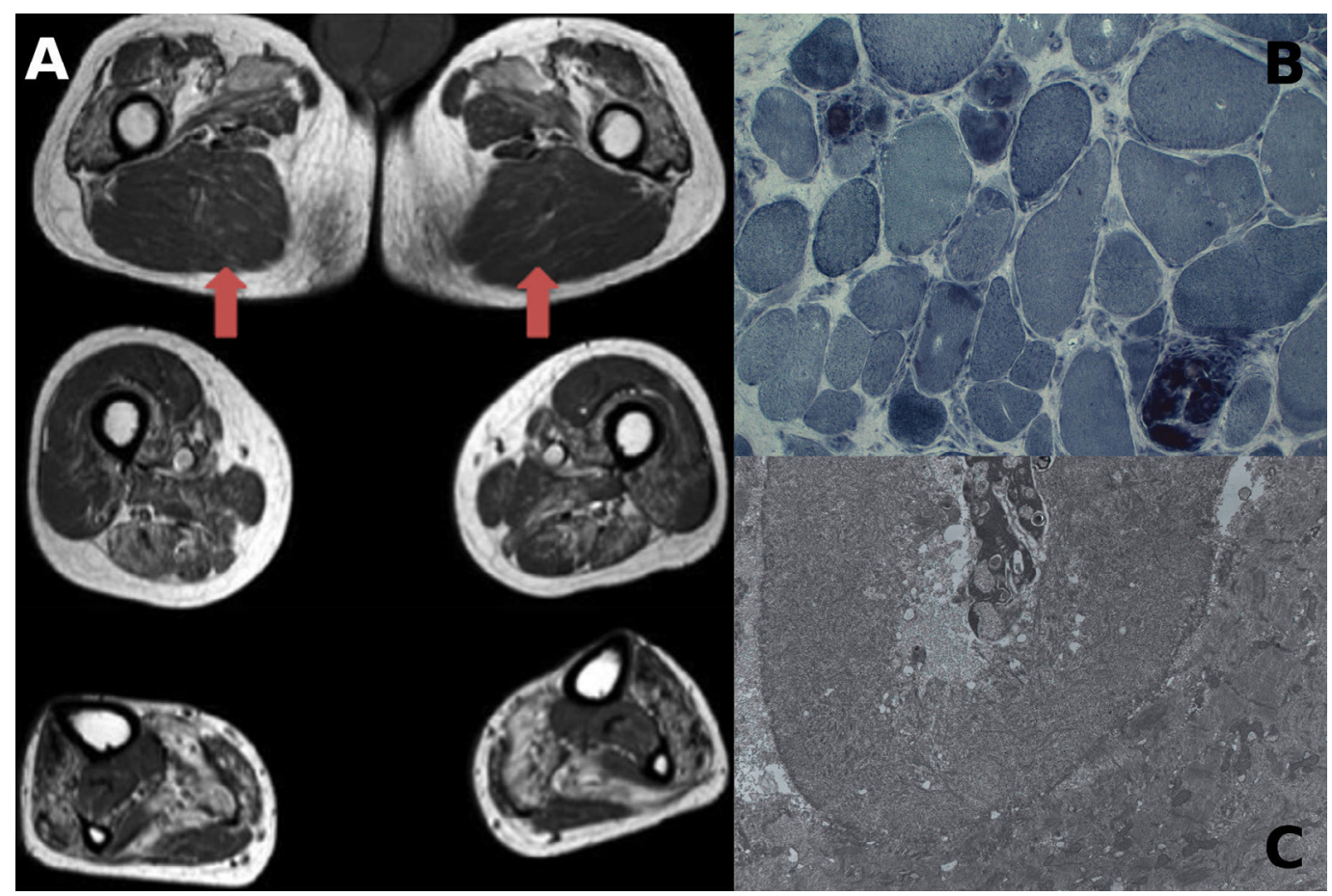

Fig. 1. (A) Lower limb muscle MRI at age 29. Striking gluteus maximus hypertrophy (arrows), fatty replacement mostly involving adductor and hamstring muscles. Soleus, medial gastrocnemius and peroneal muscles are also severely affected. (B) Muscle fibers contain darkly staining reducing bodies (menadione-NBT without substrate). (C) Electron micrograph shows a large reducing body composed of granulofilamentous material. An apoptotic nucleus is seen in the center of the body. 